\title{
DATOS ABIERTOS Y EDUCACIÓN: FORMACIÓN DE DOCENTES EN LA SOCIEDAD DIGITAL
}

\author{
Open Data and Education: Teacher Training in the Digital Society
}

\author{
María del Carmen Gálvez-de-la-Cuesta ${ }^{1}$ \\ ORCID: 0000-0002-0208-4311 \\ Manuel Gertrudix-Barrio ${ }^{2}$ \\ ORCID: 0000-0002-5869-3116 \\ Francisco García-García ${ }^{3}$ \\ ORCID: 0000-0001-5394-4804 \\ ${ }^{12}$ Universidad Rey Juan Carlos, Fuenlabrada, España \\ ${ }^{3}$ Universidad Complutense, Madrid, España \\ Correo: carmen.galvez@urjc.es
}

Recibido: $16 / 11 / 2019$

Aceptado: 02/07/2020

Resumen: El entorno educativo se enfrenta a una inevitable adaptación al ámbito digital, donde es constante el consumo acrítico de información por parte del alumnado. Garantizar un consumo crítico requiere intensificar los procesos de alfabetización mediática e informacional, ligados a la competencia digital docente, que pueden ser reforzados por el uso de productos multimedia basados en datos abiertos. La presente investigación tiene como finalidad analizar el grado de alfabetización digital y educación mediática que poseen los futuros docentes de Educación Primaria. Se adoptó una metodología de estudio de caso, a través de entrevistas semiestructuradas al profesorado encargado de formar a los futuros maestros en Educación Primaria de la Universidad de Castilla la Mancha (España). La interpretación de resultados se realizó mediante la técnica de análisis de contenido, con un modelo de revisión exhaustiva. Se concluye la necesidad de impulsar la alfabetización mediática e informacional en el ámbito de la formación docente.

Palabras clave: datos abiertos; sociedad de la información; alfabetización mediática; educación primaria; competencia digital.

\begin{abstract}
The educational environment faces an inevitable adaptation to the digital environment, where the uncritical consumption of information by students is constant. Ensuring critical consumption requires intensifying the processes of media and information literacy, linked to the digital competence of teachers, which can be reinforced by the use of multimedia products based on open data. The purpose of this research is to analyze the degree of digital literacy and media education that future teachers of Primary Education possess. A case study methodology was adopted, through semi-structured interviews with the teachers in charge of training future teachers in Primary Education at the University of Castilla la Mancha (Spain). The interpretation of results was made using the content analysis technique, with an exhaustive review model. The need to promote media and information literacy in the field of teacher training is concluded.
\end{abstract}

Keywords: open data; information society; media literacy; primary education; digital skills. 


\section{Introducción}

El presente estudio propone la creación de un modelo de análisis de parte de la formación que reciben los futuros maestros de primaria en el Grado en Educación Primaria de la Universidad de Castilla la Mancha, y más concretamente de la Mención en Tecnologías de la Información y la Comunicación en Educación (en adelante TICE). El aspecto en concreto a estudiar se incluye dentro de la alfabetización mediática e informacional, como dimensión específica del Marco de Competencia Digital Docente. En esta línea se ha analizado la percepción de los docentes que participan en la citada mención, sobre la formación que reciben los estudiantes en relación con el consumo de productos multimedia basados en datos abiertos. Este tipo de productos surge del movimiento Open Government, basado en la reutilización de información pública.

De los resultados de este análisis se concluirá si existe una clara necesidad de formar en dos vertientes. Por un lado, formación para los futuros maestros de educación primaria en un uso didáctico de productos multimedia basados en datos abiertos, que resulte práctico y crítico. Por otro lado, procesos de formación para los docentes que imparten clase en estos grados, con el fin de garantizar que sean capaces de incorporar en sus programaciones didácticas el uso de los productos señalados.

\section{Estado de la cuestión}

En el contexto actual, la formación de los docentes en el ámbito digital afronta una situación difícilmente comparable a otras. La pandemia de COVID-19 ha puesto de manifiesto las necesidades reales del profesorado frente a la educación digital y a la incertidumbre (Sánchez et al., en prensa). Esas necesidades encuentran su plasmación más clara en el nivel de competencia digital que hayan alcanzado los docentes en su proceso formativo.

Se concreta un estado de la cuestión a través de tres realidades paralelas: la competencia digital docente, los procesos de alfabetización mediática e informacional y el uso de productos multimedia informativos basados en datos abiertos. La combinación de ellas conduce a varias preguntas determinantes para la evolución de los procesos de formación del profesorado en el marco de la competencia digital docente: ¿se encuentran los docentes preparados para educar a una generación que recibe información por múltiples medios?, ¿reflejan los planes de estudio la necesidad de abordar los procesos de alfabetización mediática e informacional?, ¿hacen uso los 
docentes de productos informativos basados en datos abiertos, que puedan mejorar el consumo crítico y competente de información por parte de los estudiantes?

\section{El marco de la competencia digital docente}

La competencia digital docente ha sido definida de forma reciente, y en líneas generales, como el uso creativo, crítico y seguro de las tecnologías de información y comunicación para alcanzar los objetos relacionados con el trabajo, la empleabilidad, el aprendizaje, el tiempo libre, la inclusión y la participación en la sociedad (Instituto Nacional de Tecnologías Educativas y Formación del Profesorado, 2017). Esta caracterización del término es una evolución de la misma definición ofrecida en el año 2016 como recomendación europea, y que todavía hacía alusión al uso de ordenadores (Comisión Europea, 2005).

La aplicación de las tecnologías de la información y la comunicación (TIC) se hizo patente en España desde finales de los años 80, a través de las diferentes políticas educativas llevadas a cabo tanto desde la Unión Europea como desde el Gobierno español. Sin embargo, todas estas inversiones y esfuerzos, apoyadas también por amplios procesos formativos, no proporcionaron una solución al problema más acuciante de la integración de las TIC en el aula: el deficiente nivel de alfabetización digital y las precarias competencias en el uso y aplicación de las TIC que presentaba el profesorado de la enseñanza reglada no universitaria.

En este sentido resulta conveniente interpretar el término "alfabetización digital" en la línea de lo expresado por Cabero y Llorente (2008), como la formación de los individuos en una serie de aspectos, que incluirían desde el manejo técnico de la tecnología, el conjunto de conocimientos y habilidades que les permitan buscar, seleccionar, analizar y comprender grandes cantidades de información, la capacidad crítica ante este conocimiento y su uso en contextos cotidianos, que incluyan la expresión y comunicación con otros seres humanos. Este proceso, incluye la capacitación para determinado tipo de competencias y su gradación en niveles como los que articula el Marco de Competencia Digital Docente (Instituto Nacional de Tecnologías Educativas y Formación del Profesorado, 2017), que divide en cinco las áreas que lo componen y en seis los niveles competenciales a adquirir: 
Tabla 1.

Áreas del Marco de Competencia Digital Docente

\begin{tabular}{ll}
\hline ÁREA & NIVEL COMPETENCIAL \\
\hline Área 1 & Información y alfabetización informacional \\
Área 2 & Comunicación y colaboración \\
Área 3 & Creación de contenidos digitales \\
Área 4 & Seguridad \\
Área 5 & Resolución de problemas \\
\hline Fuente. Elaboración propia (2019)
\end{tabular}

El Plan Bolonia (European Ministers of Education, 1999) y la adaptación al Espacio Europeo de Educación Superior (EEES) permitieron numerosos cambios en la organización competencial de las titulaciones universitarias. De forma muy concreta, facilitaron la incorporación de la competencia digital como una de las ocho competencias clave que debe haberse adquirido al finalizar la enseñanza obligatoria.

\section{Alfabetización mediática e informacional en el marco de la competencia digital docente}

Intensamente relacionada con la competencia digital, surge en paralelo la necesidad de dotar de formación tanto a docentes como a estudiantes en el ámbito de la alfabetización mediática e informacional, señalada por la Unesco como una prioridad transversal (Atenas y Havemann, 2019). En torno a esta idea, autores como Tapscott (2011) señalan la evolución del modelo educativo y la transformación de un docente dueño del conocimiento en un conductor entre la desbordante información que la red hace llegar a través de múltiples medios.

Precisamente esa información se multiplicó de forma exponencial con la irrupción del social media. Redes sociales, bloggers o influencers comenzaron a tejer una amplia red informativa en una sociedad no capacitada para la discriminación de información. Esta sociedad también carecía de las competencias necesarias para ser consumidores activamente críticos, tanto en el contexto educativo como en el familiar (González-Fernández, Ramírez-García y SalcinesTalledo, 2018). De esta forma, en un entorno educativo con una mayoría del profesorado 
(universitario o no universitario) sin un nivel adecuado de competencia digital, irrumpía también la necesidad de abordar la alfabetización mediática e informacional.

El impacto social que han generado las redes sociales y la voluntad constante de compartir información que se instala en los hábitos de la ciudadanía han producido nuevos modelos comunicativos. Sin embargo, no han crecido los consumidores críticos y competentes en la discriminación y veracidad de los datos que reciben (Sanz Arazuri, Alonso Suiz, Sáenz de Jubera Ocón, Ponce de León Elizondo y Valdemoros San Emeterio, 2018).

\section{El producto multimedia basado en datos abiertos y su uso docente}

El Marco de Competencia Digital Docente da cabida en una de sus áreas a la alfabetización informacional, y una de sus competencias es la evaluación de información, datos y contenidos digitales. Esta requiere evaluar la calidad de los recursos educativos que encuentran en Internet, ser crítico con las fuentes de información y con los recursos antes de utilizarlos en el aula.

La dimensión que alcanza esta competencia puede precisarse a través del análisis de los usos que los docentes dan a determinados tipos de recursos y a su capacidad de selección entre estos. Asociado al movimiento Open Government o Gobierno Abierto, desde hace algunos años se ha puesto en marcha el desarrollo de productos informativos multimedia basados en datos abiertos u open data. Estos son datos que pueden ser utilizados, reutilizados y redistribuidos libremente por cualquier persona, y que se encuentran sujetos, cuando más, al requerimiento de atribución y de compartirse de la misma manera en que aparecen (Open Knowledge, s. f.). Se trata de datos que deben ofrecer un acceso sencillo, ser modificables de forma simple y cómoda, estar disponibles y permitir su redistribución y reutilización por cualquier ciudadano sin ningún tipo de restricción.

El sector público inició la publicación de este tipo de agrupamientos de información, tanto con la finalidad de modificar la percepción ciudadana de su misión y funciones como de facilitar los procesos de empoderamiento ciudadano (Gértrudix y Álvarez, 2014). En Estados Unidos se definen de forman precisa los conceptos, transparencia, participación y colaboración como las bases del Gobierno Abierto en el Memorando sobre transparencia y gobierno abierto firmado por el presidente Barack Obama en enero del año 2009. A partir de aquí, se inició la primera iniciativa internacional (data.gov) y posteriormente fueron surgiendo en otros países, como la del Gobierno británico (data.gov.uk) que contó con el apoyo de Tim Berners-Lee. 
Resulta de gran importancia la Declaración de Malmö del año 2009, que fijó como objetivo que "los ciudadanos y las empresas han de ser empoderados por servicios de administración electrónica [...] Para ello plantea entre sus objetivos aumentar la disponibilidad de la información del sector público para su reutilización" (Ministerio de la Presidencia, 2009). Todo este movimiento ha generado un amplio corpus normativo, del que destaca la Directiva 2003/98/CE del Parlamento Europeo y del Consejo, del 17 de noviembre de 2003. Esta se refiere a la reutilización de la información del sector público y se recoge, en el caso de España, en la Ley 37/2007 (España, 2007) de 16 de noviembre, sobre reutilización de la información del sector público.

En el año 2009, en España, se puso en marcha el Proyecto Aporta dentro del contexto del Plan Avanza 2 (2009-2012). Su objetivo más importante es promover una cultura de reutilización de la información en el ámbito de la administración pública. Así se podrá concienciar de la importancia y el valor que tiene y facilitar la puesta a disposición por parte de las administraciones y organismos públicos de la información disponible. Aporta es una iniciativa promovida por el Ministerio de Energía, Turismo y Agenda Digital, a través de la entidad pública empresarial Red.es y en colaboración con el Ministerio de Hacienda y Función Pública.

Tal y como aparece en el portal de reutilización de la información pública (datos.gob.es), nace con el objetivo de crear las condiciones para el desarrollo del mercado de la reutilización de la información del sector público, así como para dar apoyo a las unidades administrativas, en las actividades técnicas y organizativas necesarias para que publiquen de acuerdo con la legislación vigente, y de la forma más amigable para su reutilización, la información de acceso no restringido que recogen.

Por otra parte, en España, el derecho de acceso a la información pública está especialmente regulado por una ley polémica y que encontró muchas complicaciones en el momento de su publicación (Cortes Generales-Comisión Constitucional, 2013). La Ley de Transparencia y Acceso a la Información Pública tiene como misión fundamental:

Ampliar y reforzar la transparencia de la actividad pública, regular y garantizar el derecho de acceso a la información relativa a aquella actividad y establecer las obligaciones de buen gobierno que deben cumplir los responsables públicos así como las consecuencias derivadas de su cumplimiento (España, 2013). 


\section{Material y métodos}

Para la presente investigación se han tomado tres productos multimedia informativos basados en datos abiertos. Estos se mostraron a los entrevistados de forma previa, con el fin de situarles en contexto. Han sido desarrollados por instituciones públicas o entidades sin ánimo de lucro y no se incluyen dentro del modelo de REA (Recursos Educativos en Abierto), que son desarrollados específicamente para el ámbito educativo. Su misión es informar a la ciudadanía a través del uso de datos abiertos:

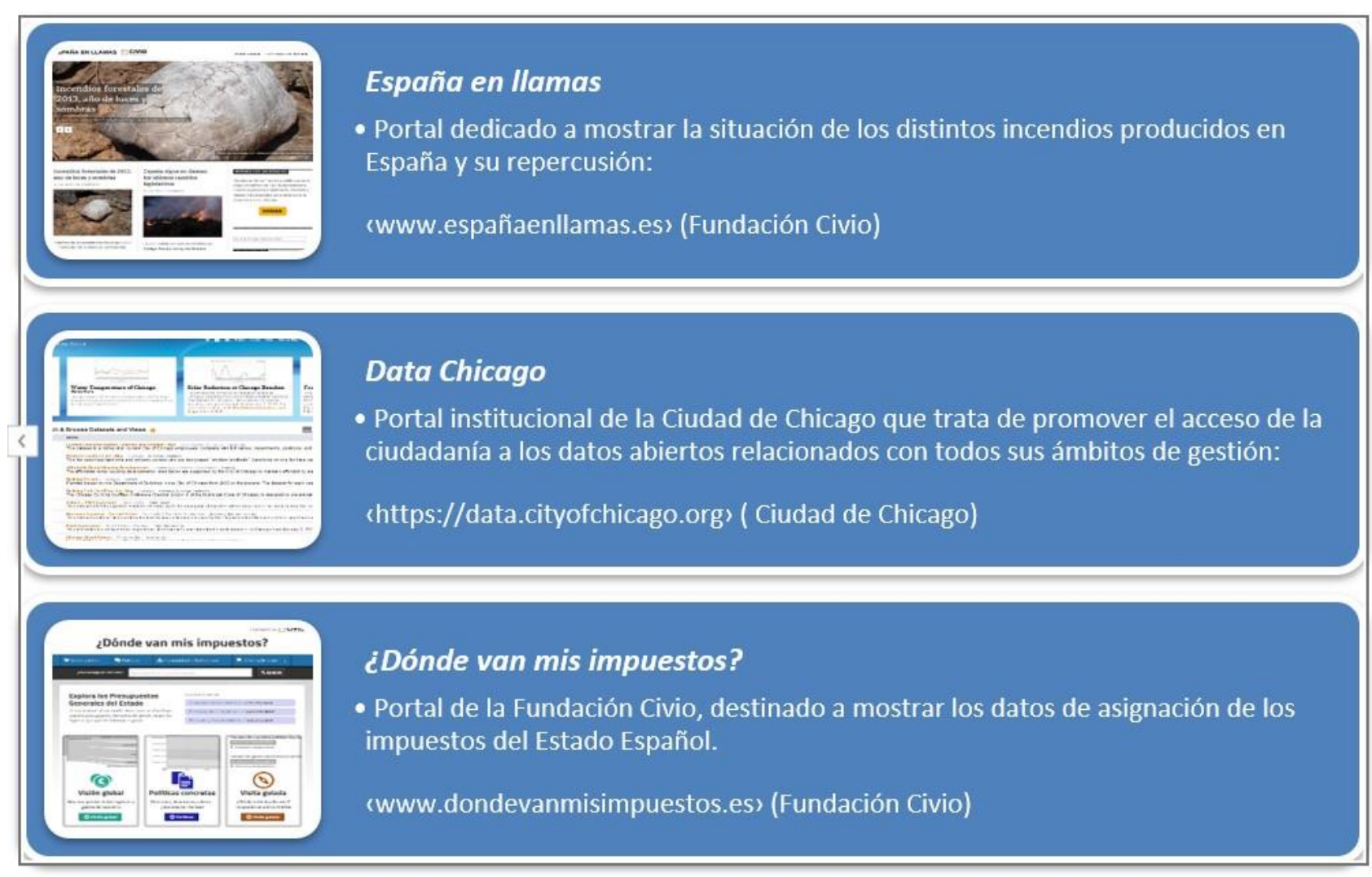

Figura 1. Productos seleccionados para el desarrollo de la investigación. Elaboración propia (2019)

\section{Diseño de la investigación}

Para la obtención de datos se realizó una serie de entrevistas a los docentes de la Mención TICE del Grado en Educación Primaria de la Universidad de Castilla la Mancha. Se trata del estudio de una realidad muy específica y concreta, enmarcado en una tipología de titulación y caracterizado por su propio contexto. Se ha llevado a cabo con la finalidad de lograr generar un 
modelo de análisis que pueda resultar trasladable a otras titulaciones. Las preguntas fundamentales de la investigación se sintetizaron en los siguientes conceptos:

- ¿ ¿Los contenidos que ofrece la Mención TICE están orientados a mejorar las capacidades de consumo de información mediática basada en datos abiertos?

- ¿ ¿La formación específica ofrecida por la Mención TICE mejora, modifica o altera de forma específica las competencias de los estudiantes en el tratamiento de la información y sus habilidades como lectores críticos, facilitando los procesos de empoderamiento ciudadano?

Con las premisas del estudio de caso y tomando como punto de referencia los principios de Yin (1994), el formato de análisis de los datos se ha desarrollado de forma cualitativa, a través del método de la entrevista. Esta fórmula genera procesos de comunicación con el sujeto entrevistado y opera y se desenvuelve en diferentes niveles de la información y la comunicación individual (Delgado y Gutiérrez, 1995).

\section{Población, marco de la muestra y proceso de selección}

Para seleccionar la muestra de docentes entrevistados se trabajó con un principio fundamental: su relación con la Mención TICE. Con este principio, se alcanzó a entrevistar a nueve sujetos que, en diferentes niveles, cumplían las siguientes condiciones:

- Ser docente en activo en la Mención TICE

- No impartir docencia en la Mención TICE, pero sí ser docente de estudiantes que están realizando la mención

- Haber impartido docencia en la Mención TICE

- Haber participado en el desarrollo y puesta en marcha de la Mención TICE

\section{Entorno del estudio}

Las entrevistas fueron efectuadas durante el mes de abril de 2013 en el Campus de Toledo de la Facultad de Educación de la Universidad de Castilla la Mancha. En todas ellas se realizó registro sonoro y fotográfico, previo consentimiento otorgado por los diferentes sujetos entrevistados. 


\section{Intervenciones}

En cuanto a las técnicas o procedimientos en recolección de datos, se facilitó a los docentes información relativa a diferentes productos informativos multimedia basados en datos abiertos (Figura 1) con el objeto de contextualizar la entrevista, así como varios descriptores relacionados con el uso de datos abiertos en el ámbito educativo. Igualmente, se les detalló en un listado las asignaturas y objetivos de la Mención TICE, para que pudiesen fundamentar adecuadamente sus respuestas. El estilo elegido para la formulación de las entrevistas fue el semiestructurado (Delgado y Gutiérrez, 1995), en torno a doce cuestiones.

\section{Análisis de los datos}

La extracción de datos de las entrevistas se realizó desde un proceso de análisis de contenido, con un modelo de revisión exhaustiva, teniendo como objetivo comprender una realidad en un contexto determinado. Se situó dentro del paradigma interpretativo (McMillan y Schumacher, 2005), buscando no solo la información, sino también su interpretación, valoración y orientación. Se trabajó también, como fuente y marco general, con el Plan de Estudios de la Mención TICE del Grado en Maestro/a en Educación Primaria. Se recuperó de este toda la información relativa a los fundamentos e implantación de la mención.

Asimismo, la tipología de conocimientos y contenidos que ofrecen los docentes a los estudiantes se contrastó con los procesos de alfabetización mediática y competencia digital que la mención TICE considera imprescindibles. De este Plan de Estudios se precisaron las asignaturas que comprendía la Mención TICE y se realizó la selección del profesorado. También se identificó si la alfabetización informacional y mediática estaba dentro de las competencias pretendidas.

\section{Análisis y resultados}

\section{Interpretación de las entrevistas}

A la hora de establecer los resultados e interpretar los datos, se generaron varias dimensiones relacionadas con las variables del estudio y con las preguntas planteadas en la entrevista. A continuación, se muestran los resultados en seis dimensiones agrupadas en función de las respuestas obtenidas. Se ilustra cada dimensión con las textualidades más significativas de los sujetos entrevistados. 
Nivel de alfabetización digital. Volumen de contenidos ofrecidos por la Mención TICE en los que se trata la competencia mediática del alumnado

En general los entrevistados manifestaron que los estudiantes adquieren un mayor grado de competencia digital al cursar la mención, dado que la formación recibida les permite adquirir una importante serie de normas, usos y códigos del lenguaje digital. Los entrevistados coinciden en que al terminar la Mención TICE, los alumnos han conseguido mejorar su nivel de competencia mediática. Finalizan la titulación con una mejor preparación para el futuro ejercicio como maestros de educación primaria, ya que conocen y han aprendido a utilizar contenidos y recursos didácticos que aparecen en la red. Igualmente opinan que se les ofrece una buena formación en herramientas básicas de investigación y análisis de la información audiovisual.

Los alumnos están en constante comunicación tanto con el equipo docente como con ellos mismos realizando trabajo colaborativo y compartiendo y debatiendo sobre informaciones educativas que sí pueden obtener a través de Internet o a cualquier otro medio de comunicación, medio de masas, medio de comunicación de masas, noticias de los telediarios, prensa digital... Cualquier medio donde ellos analizan, seleccionan, resumen, debaten noticias, se crean foros de debate para que ellos puedan participar activamente y construir entre todos pues cierto conocimiento siempre desde una versión crítica y con la mediación y la supervisión del equipo docente, claro (Entrevistado 1).

Nivel de conocimiento ciudadano acerca de la oferta de información basada en datos abiertos. Información que ofrecen los productos informativos multimedia basados en datos abiertos. Volumen o cantidad de contenidos ofertados por la Mención TICE, en los que se trata la competencia mediática del alumnado en el consumo de datos abiertos.

Se considera que en esta especialidad no se tratan de forma adecuada los productos multimedia informativos basados en datos abiertos, en buena medida debido al desconocimiento que los docentes presentan de este tipo de contenido. Manifiestan que, a pesar del escaso uso de estos productos, la formación en su aplicación en el aula debería ser un elemento más tenido en cuenta en el ejercicio docente, ya que aportan información, datos valiosos y fiables y, sobre todo, facilitan la generación de un espíritu crítico y competente en el consumo de medios, indispensable para un futuro maestro en educación primaria. Reconocen que la formación de formadores en la utilización de productos informativos multimedia basados en datos abiertos permitiría enseñarles a diferenciar la información real de las fake news (Fernández-García, 2017). 
Comentándolo con los compañeros, algunos, por ejemplo, cuando hablas sobre el tema de alguna aplicación con... con "datos abiertos" como puede ser, por ejemplo, Infocarreteras, que es un servicio público que te da a conocer el estado de la situación de las carreteras; hay también temas de salud con el tema de la contaminación para enfermos que sepan el nivel de contaminación cuando vayan a... a salir de paseo. Y yo creo que ese, este tema no se trata bastante en la universidad. No como se debiera. No creo que haya un... por lo menos que sí hay al respecto no van en esa dirección (Entrevistado 2).

Competencias específicas contempladas en la Mención TICE sobre la formación del alumnado en consumo crítico de medios de información.

Los entrevistados coinciden en que se ofrece una formación tecnológica necesaria en el desarrollo de las competencias de los futuros maestros, la que les proporcionaría una dimensión comunicativa. Existe una clara intención por transmitirles la inquietud por el conocimiento de la verdadera naturaleza de las cosas y el análisis objetivo de la realidad. Es especialmente representativa la alusión a la competencia mediática de los docentes universitarios sobre este aspecto. Consideran que necesitan más formación para mejorar el propio uso de herramientas adecuadas, que les permitirían educar a los alumnos en nuevos usos didácticos y en la adquisición de competencias para discernir toda la información que reciben.

Bien, en ese sentido creo que es más complicado trabajar esta competencia en la Mención TICE. ¿Por qué? Porque creo que aún, incluso los propios docentes, nos falta formación en este sentido. Lo de desarrollar el juicio crítico, la capacidad crítica, discernir eh... las informaciones a tratar desde un punto de vista selectivo, objetivo... nos cuesta todavía a todos. Entonces, creo que ahí sería una de las competencias quizás donde flojearíamos, digamos, donde quizás tuviéramos que incidir más en el trabajo con los alumnos porque es complicado (Entrevistado 2).

\section{Grado de competencias mediáticas adquiridas a través de aprendizaje informal}

Existe una amplia coincidencia entre todos los docentes al tratar esta dimensión. Señalan que el nivel de competencia mediática de sus alumnos está más basado en aprendizajes informales que en los procesos formativos que se les ofrecen desde la propia Mención TICE. Opinan que como miembros de la generación definida como nativos digitales (Prensky, 2001), acceden de una 
forma activa a la información a través del ámbito digital, si bien lo hacen especialmente por vías informales. A pesar de esto, carecen de conocimientos previos en el tratamiento, localización y contraste de la información, que por el momento no les es transmitido desde sus estudios de Grado.

Entonces, yo creo que sí, de forma informal aprenden mucho y saben mucho y comparten mucha información y están en muchas redes sociales, pero quizás falle lo que aporta la educación formal, en este caso lo que, podíamos decir, lo que aporta la Mención TICE. Es decir, darle un poco de sentido crítico, de sentido selectivo a toda esa información informal o aprendizaje informal con lo que ellos acuden a la Mención TICE (Entrevistado 1).

Grado declarativo de los contenidos de la Mención TICE sobre la influencia del aprendizaje informal del alumnado en los procesos de consumo de medios de comunicación

Esta dimensión ofrece, a diferencia del resto, posiciones disociadas entre los docentes entrevistados. Por una parte, un grupo de docentes considera que la Mención TICE sí tiene en cuenta el nivel de competencia mediática que los estudiantes han adquirido por medios informales. Igualmente opinan que esta forma de aprendizaje es totalmente necesaria y asociada al aprendizaje formal. Por otro lado, otro grupo de profesores cree que durante el desarrollo curricular de la Mención no se ha tenido en cuenta el conocimiento que los estudiantes habrían podido adquirir de manera informal. Igualmente consideran que los procesos están mucho más orientados al uso de recursos tecnológicos en el aula, sin consensuar previamente los diferentes niveles de competencia mediática que puedan presentarse entre los discentes. Finalmente, existe un bloque que opina que, aunque tratan de considerar el nivel de competencia digital adquirido desde el aprendizaje informal, no pueden asegurar que se logre trasladar al aula y a la concreción de contenidos.

Realmente establecemos que el aula no es algo cerrado sino que es de alguna manera un marco abierto donde los alumnos también ingresan el conocimiento desde otras fuentes, desde los medios de comunicación. De hecho además tienen experiencia, o sea, ellos también a través de nuestro propio periódico digital, o la radio salen... es decir, abrimos un poquito una ventana continua para que los alumnos desde fuera también aporten (Entrevistado 9). 
Grado declarativo del nivel de preparación/capacitación del alumnado del Grado en Maestro/a en Educación Primaria para instruir a sus futuros alumnos en el consumo crítico y responsable de productos mediáticos preferentemente basados en datos abiertos

La opinión generalizada de los docentes es que la formación que se ofrece a los estudiantes de la Mención TICE será suficiente para que inicien su andadura profesional con un componente de sensibilización hacia un consumo responsable de la información, pero dudan de si serán capaces de transmitir estos valores. Por otro lado, creen que en su futuro como docentes no encontrarán suficientes herramientas en el aula que puedan permitirles trabajar con este tipo de productos. Esto dificultará que puedan aplicar sus posibilidades a la formación de su alumnado en competencias mediáticas. Se emite también, como resultado paralelo de este análisis cualitativo, una respuesta unánime en todos los entrevistados en cuanto a la creación de la Mención TICE: la resistencia de una gran parte del profesorado al empleo de nuevos métodos docentes y a la vinculación con las TIC de los procesos en general.

Cuando tú vas a los colegios, todo lo que tú puedas aquí ver no está allí porque los recursos TICE que hay en los colegios son tan nimios, son tan pobres que no te dan pie a siquiera... Puedes plantear esa actividad pero para llevarla a cabo te va a costar muchísimo (Entrevistado 2).

\section{Interpretación de resultados}

Una vez llevado a cabo el análisis del contenido de las entrevistas de forma cualitativa, aparece una idea clara. En general, el profesorado de la Mención TICE desconoce en buena medida las posibilidades que en el ámbito del desarrollo de la competencia informacional y mediática pueden ofrecer los productos informativos multimedia basados en datos abiertos.

Resulta también evidente una cierta prudencia entre los docentes sobre si los contenidos que ofrece la Mención permiten obtener suficientes capacidades en el consumo de información mediática. En la década anterior, Cabero (2007) o Pérez-Tornero (2009) ya señalaban que la alfabetización mediática no era un concepto nuevo, sino una necesidad surgida en los primeros años de la televisión. Por esta razón, las competencias de los docentes en este sentido deberían estar garantizadas en una sociedad con niveles de consumo mediático muy elevado.

Igualmente, en este sentido manifiestan escasa confianza en las futuras opciones que tendrán sus alumnos como maestros de Educación Primaria, al intentar aplicar los conocimientos 
recibidos en el uso responsable y crítico de la información basada en datos abiertos. Precisamente la actitud favorable de los docentes que se acercan a las TIC es considerada un factor indispensable (Torres, 2011) y, junto con la formación digital, sería un elemento de primer orden para mejorar su relación con el ámbito digital (Marqués, 2008).

Ahondando en la competencia mediática como valor imprescindible de la competencia digital docente, las entrevistas han dejado también de manifiesto cómo los docentes no dudan en afirmar que la Mención TICE mejora las capacidades de los estudiantes en el tratamiento de la información, y en sus posibilidades de ser críticos y selectivos. No obstante, manifiestan cierto escepticismo sobre si el uso de este tipo de productos influya en conseguir unos niveles óptimos de empoderamiento ciudadano, en contraposición a la teoría de Carlsson, Tayie, JacquinotDelaunay y Tornedo (2008). Los mencionados autores señalan precisamente que la capacidad para ejercer de una manera crítica la lectura de estos productos, así como la competencia para comprender su significado, es la que podría conseguir que se conformase una ciudadanía activa y participativa. Igualmente podría alcanzar cuotas destacadas de empoderamiento ciudadano.

Existe también diversidad de opiniones en el ámbito de la influencia generada por el aprendizaje informal como agente catalizador de los procesos de adquisición de competencias digitales. Esta diferencia de pensamiento oscila entre tres elementos: por un lado, la creencia de que el aprendizaje informal es imprescindible para garantizar que los alumnos obtengan unos niveles de competencia digital óptimos y, por tanto, capacidades suficientes para una selección y consumo crítico de información; por otro lado, la idea de que el aprendizaje informal no es tenido en cuenta en el desarrollo curricular de la Mención TICE y por ende no es preceptivo considerarlo como un elemento que tenga efectos reales y medibles; y finalmente, la afirmación de que a pesar de que el aprendizaje informal es tenido en cuenta en el desarrollo curricular de la Mención, existe un desconocimiento completo sobre la forma en que los estudiantes obtienen realmente niveles adecuados de competencia digital y alfabetización mediática.

En general, y como elemento destacable de esta interpretación, existe también una tendencia a confundir este tipo de productos con otros desarrollados en código abierto o libres de derechos, pero no generados a través de datos abiertos. Igualmente, varios de los docentes desconocen el concepto de "competencia digital docente", y lo asocian mucho más con criterios de "informática educativa" o "uso de herramientas tecnológicas", lo que retrotrae a períodos anteriores en todo lo referente a la aplicación de las TIC en la educación. 
También se enmarca en la línea de lo expresado por Buckingham (2011), quien señala que existe una visión de tipo reduccionista, que limita la importancia de la alfabetización mediática al conocimiento de las herramientas tecnológicas que permiten acceder a la información y no al análisis y consumo crítico de esta. Resulta también destacable que, a pesar de la falta de acercamiento a este tipo de productos, todos los entrevistados entienden como fundamental el iniciar políticas de alfabetización mediática. Estas deberían incluir objetivos más directos en las titulaciones que forman a los futuros docentes, que obliguen a generar nuevo contenido y nuevos desarrollos curriculares a los responsables de la formación universitaria.

\section{Discusión y conclusiones}

El objeto fundamental de esta investigación es el análisis de las competencias digitales y el nivel de educación mediática de los futuros maestros de Educación Primaria, relacionadas con el conocimiento, los procesos de búsqueda, selección, tratamiento, análisis y transferencia, y la actitud ante información multimedia e interactiva basada en datos abiertos. Aunque se trata de un caso específico, sí se puede realizar una aproximación a la situación real de la competencia mediática y su reflejo en la competencia digital docente en estas titulaciones, de mano de sus propios docentes.

Desde el punto de vista normativo, la Mención TICE realiza un esfuerzo considerable en detallar los diferentes procesos de alfabetización digital que permitirán a los estudiantes del Grado en Maestro/a en Educación Primaria alcanzar un nivel de competencia digital suficiente y que garantice una práctica óptima en su ejercicio docente. A este mismo nivel, se integra en el currículo la necesidad de dotar a los estudiantes de capacidad crítica. Igualmente se identifica la necesidad de formarlos en alcanzar habilidades que les faculten para la búsqueda, obtención, procesamiento, y comunicación de la información de manera competente y que, a su vez, puedan llegar a transformar en conocimiento.

En cuanto al conocimiento que los estudiantes demuestran de los productos multimedia basados en datos abiertos, y la obtención de estos tanto a través de medios formales como informales, resulta evidente que los docentes declaran que no se realiza un uso habitual de estos en el aula. Por tanto, no se puede considerar la vía formal como una fuente efectiva. Sin embargo, sí es especialmente destacable la diferenciación entre los objetivos de la Mención TICE y el resto de las menciones, en lo relativo a la potenciación del espíritu crítico del alumnado, dirigido especialmente al uso y consumo de determinado tipo de recursos audiovisuales. A pesar de ello, 
la Mención TICE no se ocupa de forma específica de los productos que se elaboran a través de datos abiertos, ni desde el punto de vista curricular ni desde las actuaciones y actividades de aula que llevan a cabo los docentes.

Es destacable, por otra parte, el escepticismo que los entrevistados manifiestan en cuanto a la posibilidad de la formación en el uso de productos informativos multimedia basados en datos abiertos. Dudan de que su culminación en adquisición de niveles óptimos de competencia mediática germine en procesos de empoderamiento ciudadano, puesto que, en su opinión, se generaría un tipo de empoderamiento virtual que no redundaría ni respondería a un proceso real que se viese reflejado en la sociedad.

Emana como conclusión de este modelo de análisis que la Mención TICE es una especialidad destinada a ofrecer un alto nivel de competencias en la integración de las TIC en el aula. El planteamiento general de la mención está constituido alrededor de la competencia digital, y trata de ayudar a sus estudiantes a conseguir unos niveles adecuados que les garanticen independencia en su labor docente y proactividad en la integración de las TIC en el aula.

Resulta, por otra parte, una titulación que realiza un importante desglose de competencias orientadas hacia el fomento de la competencia mediática del alumnado y preocupada por promover un espíritu democrático y las suficientes capacidades para incrementar actividad y participación ciudadana. Desde la perspectiva de los datos abiertos, y con la seguridad de que la transparencia pública y la información deben contar con un espacio suficiente dentro de la Educación Superior, no se puede olvidar que el alumnado debe recibir formación integral para ser competentes en el análisis de sus propias estructuras mentales en relación con la realidad social, económica o educativa. En este caso, la Mención TICE no recoge estas posibilidades ni las trata en su desarrollo.

Es muy significativo, como se indicaba en la interpretación de los resultados, que los docentes se demuestren escépticos y preocupados ante la puesta en marcha de la actividad docente de sus alumnos, cuando en un futuro ocupen su papel como maestros/as en Educación Primaria. Consideran que existirán grandes carencias que no les permitirán integrar con fluidez las tecnologías en el aula, así como una importante falta de apoyo desde los equipos docentes.

Los resultados y conclusiones ofrecidos a través del presente estudio de caso se muestran vigentes en el estudio realizado por Rivas-Rebaque, Gértrudix-Barrio y Cisneros de Britto (2019) en las Facultades de Educación españolas. En este estudio se consultaba a los docentes sobre el 
conocimiento y uso de los datos abiertos, y un alto porcentaje (89\%) manifestó la necesidad de formación tanto específica como transversal a la hora de utilizarlos en el ámbito educativo.

Por el momento, el panorama general muestra un contexto en el que han aparecido iniciativas claras que tienen como misión el impulso de la competencia digital docente a diferentes escalas, como la señalada en la introducción de este estudio y llevada a cabo por el Instituto Nacional de Tecnologías Educativas y Formación del Profesorado (INTEF; 2017). Está teniendo diversa repercusión en niveles de educación no universitaria, pero está encontrando un eco importante en el marco universitario. Este eco se manifiesta fundamentalmente en la puesta en marcha de diferentes estudios de postgrado enfocados a la formación de docentes no universitarios en el ámbito de la competencia digital docente (Universidad Rey Juan Carlos, Universidad Nacional de Educación a Distancia) y en iniciativas (Carrera y Coiduras, 2012) que comienzan a considerar esta perspectiva como ineludible en los procesos de transformación digital de la Educación Superior.

No cabe duda de que la situación derivada de la pandemia de COVID-19 ahonda especialmente en estas iniciativas y en una necesidad ingente para todas las modalidades de aprendizaje. Vincular estas iniciativas con el impulso del conocimiento que el uso y aplicación de los datos abiertos puede ejercer sobre la educación apoyaría de forma indudable la consecución de unos niveles aceptables de competencia mediática e información en el alumnado. El hilo conductor sería la competencia digital docente, elemento imprescindible para garantizar una formación adecuada en la ciudadanía, libre de elegir diferentes tipos de informaciones, diferenciarlas y realizar un consumo crítico e independiente.

Como elemento final de la discusión, y continuación de la estructura expuesta en la introducción a este texto, se retoman sus tres líneas fundamentales: la competencia digital docente, la competencia mediática e informacional y los datos abiertos. Más allá de las posibles dificultades técnicas o relacionadas con la posible obsolescencia de las infraestructuras tecnológicas, el principal problema para el desarrollo de la competencia digital docente continúa siendo el nivel de capacitación del profesorado. La formación de los futuros docentes es el único elemento que puede garantizar que los responsables de la educación de las próximas generaciones de ciudadanos dominen el uso de las TIC en el aula, con capacidad de adaptación a los procesos digitales y con competencias suficientes para seguir sin problemas la evolución del entorno. 
Sobre la competencia mediática, y siguiendo a Bauman (2007), los medios de comunicación, en todas sus manifestaciones, habrán de progresar en el marco tecnológico, al tiempo que se adaptan a las necesidades de los usuarios con efectividad e inmediatez.

Por último, el uso y difusión de datos abiertos es una herramienta indispensable para canalizar información real, verídica y especialmente adecuada para organizar y sostener la estructura de una ciudadanía digital destinada a alcanzar elevados niveles de empoderamiento ciudadano (Linarez, 2016).

En el marco de ese horizonte futuro se sitúan las acciones llevadas a cabo en el proyecto Ciudadanía Digital y Open Data Access: empoderamiento ciudadano a través de los medios sociales en el entorno digital del Ministerio de Economía y Competitividad, y que ha tenido continuación en el proyecto que financia esta investigación y en la iniciativa Escuelas Comciencia. Esta se centra en el acercamiento de la ciencia a la educación y se preocupa por el desarrollo del pensamiento crítico y reflexivo a través de la educación basada en datos abiertos y la aplicación del método científico. Escuelas Comciencia trata de contribuir a la formación de los futuros ciudadanos con varios objetivos: la reflexión sobre el conocimiento científico-tecnológico, la alfabetización digital y la adquisición de competencias y habilidades transversales que permitan a los estudiantes una participación activa en la vida social, el uso de datos abiertos y el desarrollo del pensamiento razonado y libremente elegido.

\section{Referencias}

Atenas, J., y Havemann, L. (2019). Open data and education. En T. Davies, S. Walker, M. Rubinstein, y F. Perini (Eds.), The state of open data: Histories and horizons (pp. 91-102). Cape Town/Ottawa: African Minds and International Development Research Centre. https://doi.org/10.5281/zenodo.2677851

Bauman, Z. (2007). Los retos de la educación en la modernidad líquida. Barcelona, España: Gedisa.

Buckingham, D. (2001, junio). Media Literacy: new directions or losing our way?. Presentado en Manifesto for Media Education Symposium. Royal Institute of British Architects, Londres, Reino Unido.

Cabero, J. (2007). Tecnología educativa: su evolución histórica y su conceptualización. En J. Cabero (Ed.), Tecnología Educativa (pp. 13-28). Madrid, España. Mc. Graw-Hill.

Cabero, J., y Llorente, M. C. (2008). La alfabetización digital de los alumnos. Competencias digitales para el siglo XX. Revista Portuguesa de Pedagogía, 42(2) 7-28.

Carlsson, U., Tayie, S., Jacquinot-Delaunay, G., Pérez Tornero, J. M. (2008). Empowerment Through Media Education: An Intercultural Dialogue. Media International Australia, 129(1) 154-155. 
Carrera, F., y Coiduras, J. (2012). Identificación de la competencia digital del profesor universitario: un estudio exploratorio en el ámbito de las Ciencias Sociales. REDU Revista de Docencia Universitaria, 10(2), 273298. Recuperado de https://repositori.udl.cat/bitstream/handle/10459.1/47980/018608.pdf?sequence=1

Comisión Europea. (2005). Propuesta de Recomendación del Parlamento Europeo y del Consejo sobre las competencias clave para el aprendizaje permanente. Bruselas. Recuperado de https://www.europarl.europa.eu/meetdocs/2004_2009/documents/com/com_com(2005)0548_/com_com(20 05)0548_es.pdf

Cortes Generales-Comisión Constitucional. (2013). Comparecencia de don Manuel Sánchez de Diego y Fernández de la Riva, Profesor titular de Derecho de la Información de la Universidad Complutense de Madrid, acordada por la citada Comisión, para informar en relación con el Proyecto de Ley de transparencia. Diario de Sesiones Del Congreso de Los Diputados, X Legislat, 25-28. Recuperado de http://www.congreso.es/public_oficiales/L10/CONG/DS/CO/DSCD-10-CO-254.PDF\#page=17

Ministerio de la Presidencia. (2009). Declaración Ministerial sobre la administración electrónica. Adoptada de forma unánime en Malmö, Suecia, el 18 de noviembre de 2009. Recuperado de https://administracionelectronica.gob.es/pae_Home/dam/jcr:e7e0d4ca-e3ac-41a7-a0d5ca80bc122639/BIBLIOTECA_PUBLICACIONES_PE_2010_Traduccion_castellano_Declaracion_Ministe rial_de_Malmo.pdf

Delgado, J. M., y Gutiérrez J. (1995). Métodos y Técnicas Cualitativas de Investigación en Ciencias Sociales. Madrid, España: Síntesis.

España. (2007). Ley 37/2007, de 16 de noviembre, sobre reutilización de la información del sector público. Boletín Oficial Del Estado, de 17 de Noviembre de 2007, núm. 276, 47160-47165. Recuperado de http://www.boe.es/boe/dias/2007/11/17/pdfs/A47160-47165.pdf

España. (2013). Ley 19/2013, de 9 de diciembre, de transparencia, acceso a la información pública y buen gobierno. Boletín Oficial Del Estado, de 10 Diciembre de 2013, núm. 295, 97922-97952. Recuperado de http://www.congreso.es/constitucion/ficheros/leyes_espa/1_019_2013.pdf

European Ministers of Education. (1999). The Bologna Declaration of 19 June 1999. Recuperado de http://www.ehea.info/article-details.aspx?ArticleId=43

Fernández-García, N. (2017). Fake news: una oportunidad para la alfabetización mediática. Nueva Sociedad, 269, 6677. Recuperado de https://nuso.org/media/articles/downloads/5.TC_Fernandez_269.pdf

Gértrudix, M., y Álvarez, S. (2014). Las Fases del Empoderamiento. https://doi.org/10.6084/m9.figshare.1092528

González-Fernández, N., Ramírez-García, A., y Salcines-Talledo, I. (2018). Competencia mediática y necesidades de alfabetización audiovisual de docentes y familias españolas. Educación XX1, 21(2). https://doi.org/10.5944/educxx1.16384

Instituto Nacional de Tecnologías Educativas y Formación del Profesorado. (2017). Marco Común de Competencia Digital Docente Octubre 2017. Recuperado de http://aprende.educalab.es/wpcontent/uploads/2017/11/2017_1020_Marco-Común-de-Competencia-Digital-Docente.pdf 
Linarez, G. (2016). El empoderamiento ciudadano del siglo XXI y las TIC. PAG. Revista Iberoamericana de Producción Académica y Gestión Educativa, 3(5). Recuperado de https://www.pag.org.mx/index.php/PAG/article/view/404

McMillan, J., y Schumacher, S. (2005). Investigación educativa. Una introducción conceptual. Madrid, España: Pearson.

Open Knowledge. (s. f.). Open Data Handbook. Recuperado de http://opendatahandbook.org/guide/es/what-is-opendata/

Pérez-Tornero, J. (2009). El nuevo horizonte europeo de la alfabetización mediática. Telos: Cuadernos de comunicación e innovación, 79, 6-7.

Prensky, M. (2001). Digital Natives, Digital Immigrants. Recuperado de http://www.marcprensky.com/writing/Prensky

Rivas-Rebaque, B.; Gértrudix-Barrio, F. \& Cisneros de Britto, J. C. (2019). The perception of the University Professor regarding the use and value of Open Data. Educación XX1, 22(2), 141-163. https://doi.org/10.5944/educXX1.21317

Sánchez, M., Martínez, A. M., Torres, R., De Agüero, M., Hernández, A., Benavides, M., Jaimes, C., y Rendón, V. (en prensa). Retos educativos durante la pandemia de COVID-19: una encuesta a profesores de la UNAM. Revista Digital Universitaria. Recuperado de https://www.revista.unam.mx/prensa/retos-educativosdurante-la-pandemia-de-covid-19-una-encuesta-a-profesores-de-la-unam/

Sanz Arazuri, E., Alonso Suiz, R.A., Sáenz de Jubera Ocón, M., Ponce de León Elizondo, A., y Valdemoros San Emeterio, M. A. (2018.). Ocio, redes sociales y estudiantes españoles. Educación XX1, 21(2). https://doi.org/10.5944/educxx1.19538

Tapscott, D. (2009). La era digital. Cómo la generación net está transformando al mundo. México D. F., México: McGraw Hill.

Torres, G. (2011). Alfabetización digital y actitudes hacia las TIC (Tesis Doctoral). Universitat Autònoma de Barcelona. Facultad de Ciencias de la Educación. Recuperado de https://www.educacion.gob.es/teseo/imprimirFicheroTesis.do?idFichero=uNXDJFIUkZk\%3D

Yin, R. K. (1994). Case Study Research: Design and Methods. Thousand Oaks, CA: Sage.

\section{Financiamiento}

Este artículo es producto del proyecto de Investigación titulado "COMCIENCIA. Comunicación eficaz, eficiente y responsable para proyectos de investigación competitivos", referencia CSO2017-82875-C2-1-R, financiado por la Dirección General de Investigación y Gestión del Plan Nacional de I+D+i, del Ministerio de Ciencia e Innovación, dentro del Observatorio de Comunicación científica de la Universidad Rey Juan Carlos.

\section{Contribución autoral}

a) Concepción y diseño del trabajo; b) Adquisición de datos; c) Análisis e interpretación de datos; d) Redacción del manuscrito; e) revisión crítica del manuscrito.

K. S. ha contribuido en a, b, c, d, e.

Editora científica responsable: Mag. Florencia de León 